\title{
Market liquidity and capital structure: Does it really matter for Vietnam's family ownership?
}

Kien Son Nguyen ( $\nabla$ kien.ns1990@gmail.com )

Monash University https://orcid.org/0000-0001-9192-1195

Van Thi Hong Nguyen

Vietnam Netherlands Program

Hang Thu Le

Eastern International University

Original Research

Keywords: capital structure, family ownership, stock market liquidity

Posted Date: February 17th, 2021

DOI: https://doi.org/10.21203/rs.3.rs-201538/v1

License: (1) This work is licensed under a Creative Commons Attribution 4.0 International License.

Read Full License 


\title{
Market liquidity and capital structure: Does it really matter for Vietnam's family ownership?
}

\author{
Kien Son Nguyen ${ }^{a, b}$ \\ Van Thi Hong Nguyen ${ }^{c}$ \\ Hang Thu Le d $^{\text {Han }}$
}

${ }^{a}$ Department of Economics, Monash University, Australia. Email: son.nguyen1@monash.edu

${ }^{b}$ Department of Economics, The University of Western Australia.

c Vietnam Netherlands Program, Vietnam. Email: van.nth@vnp.edu.vn

${ }^{d}$ Becamex Business School, Eastern International University, Vietnam. Email: hang.le@eiu.edu.vn 


\section{Declarations}

\section{Authors' contributions and confirmations}

The author Kien S.N. performed the ideas, conceived the conceptual framework, interpreted the results, structure the data, and wrote the manuscript. The author Van T.H.N. provided empirical review, studied research background and research framework, and supervised the data analysis. The author Hang T.L. studied the literature review, collected data, and provided the initial stage of data analysis.

\section{Funding}

This research did not receive any specific grant from funding agencies in the public, commercial, or not-for-profit sectors.

\section{Availability of data and materials}

All data of all listed firms are gathered via Vietnam's stock exchange - Ho Chi Minh Stock Exchange (HOSE) from Thomson Reuter Eikon data sources by yearly estimation. The data analysis and research models are conducted through software including MS Excel and STATA. Henceforth, all data and materials have been available upon the enquiry.

\section{Competing interests}

The authors declare that they have no competing interests. The authors also confirm that the content of the manuscript has not been published, or submitted for publication elsewhere. 


\title{
Market liquidity and capital structure: Does it really matter for Vietnam's family ownership?
}

\begin{abstract}
This study analyzes the moderating effect of different kinds of family ownership on the relationship between market liquidity and capital structure. Using a new sample of 315 Vietnam listed firms for five years, we figured out a significant negative link between stock market liquidity and capital structure. However, it is well noted that there is an adverse reaction from family ownership where the higher the dual-class control and dynamic structure mechanism, the more control-enhancement the family ownership will be, which leads to a higher risk aversion regarding debt-bankruptcy. In this sense, corporate leverage had responded positively to an increase in stock liquidity in the case of family ownership intervention.
\end{abstract}

Keywords: capital structure, family ownership, stock market liquidity

JEL classification: G10; G31; G32 


\section{Introduction}

Ever since the paper by Modigliani and Miller (1958) was published, market liquidity has been posited as one of the most dominant factors determining the leverage ratio (Butler, Grullon, \& Weston, 2005; Hennessy \& Whited, 2005; Lipson \& Mortal, 2009). The literature, empirically, has confirmed the profound impact of stock liquidity on the corporate leverage for different contexts from the developed markets (Hennessy \& Whited, 2005; Lipson \& Mortal, 2009; Sharma \& Paul, 2015) to the developing markets such as Thailand (Udomsirikul, Jumreornvong, \& Jiraporn, 2011), or Vietnam (Thanh \& Mai, 2017; Vo, 2017). However, there is a challenging question from the "agency" theory revealed the contradict benefits among different groups of ownership that the concentrated ownership patterns would potentially subsume the effects of other factors on capital structure choice of the firms (Deesomsak, Paudyal, \& Pescetto, 2004; Sharma \& Paul, 2015). Furthermore, most of the literature on the determinants of capital structure was ample but inclusive and neglect the role of ownership intervention, especially in the case of family ownership. Family members scared of losing their control may try to reinforce their power and would potentially overwhelm the main effects of market liquidity toward capital structure so that they preferred more debt than outside sources (C éspedes, González, \& Molina, 2010; King \& Santor, 2008; Setia - Atmaja, Tanewski, \& Skully, 2009; Sharma \& Paul, 2015).

Our study overcomes this gap by examining the interaction effects of family ownership and market liquidity on capital structure, and it is the first attempt to consider these effects in the context of one emerging country, namely Vietnam. Empirically, Duc and Tri (2014), Kien and Duc (2015) , Kien (2020) has emphasized, as there is the presence of "divided powers" and "gap power" from the group of concentrated holders among Vietnam listed company. We address this literature gap by constructing the new data set of Vietnam's family ownership (a sample of 315 listed firms during five years sample from 2013 to 2017), and demonstrating the potential effects of family power on the relationship between market liquidity and capital structure. It is worth that this study can be one of the first of its kind in current literature analyzing the interaction effect of stock liquidity and the family's ownership on capital structure, especially for the ASEAN region. In addition, this paper, exclusively for the capital structure, is profoundly

different from all previous studies of determinants of capital structure in Vietnam's market (Dang, Ly Ho, Dzung Lam, Thao Tran, \& Vo, 2019; Moussa \& Elgiziry, 2019; Thanh \& 
Mai, 2017; Tran, Hoang, \& Tran, 2018) where it employed several measurements of three types of market liquidity, two proxies of leverage, and various dimensions of family controls to secure the results and robustness to robust results for the analysis. Lastly, the panel data sample's potential bias will be solved efficiently by using a new modern technique of panel quantile regression.

\section{Market liquidity, capital structure and family ownership}

The relationship between stock liquidity and capital structure has been extensively studied, primarily progressed by Modigliani and Miller (1958). Miller and Rock (1985) and Lev (1988) suggested that the link between the asymmetric information and security characteristic has to lead the corporate capital structure to adverse selections, includes a higher transaction cost and a lower volume of trades. This, in turn, induces administrators to use leverage to signal information for future earning of firms (Miller \& Rock, 1985). Given the increasing attention to liquidity within the characteristic of asymmetric information, it is also vital to clarify more on the Pecking Order theory by Myers and Majluf (1984). Firms will tend to depend firstly on their possible earnings, next on debt sources, and finally on equity financing. At this time, the noticeable increase of market liquidity can lower the cost of equity and would lead equity to be more attractive than debt, which will decrease the percentage of debt in the corporate capital structure (Udomsirikul et al., 2011). As a result, under the mechanism of asymmetric information, the Pecking Order preference and the trade-off of the costs of debt would motivate the firm to issue equity when there is higher liquidity instead of debt (ElBannan, 2017; Fama \& French, 2002). , Frieder and Martell (2006), Lipson and Mortal (2009), and Kryzanowski, Lazrak, and Rakita (2010) tested the relationship between stock market liquidity and corporate capital choice, agreed that there is a significant negative effect of the level of security liquidity toward firm leverage. This revealed that firms with higher stock liquidity could lessen the reliance on the equity cost.

Despite the growing interest in how market liquidity links to the leverage ratio, there is another problem altering the corporate capital structure. It is well established that the concentrated ownership patterns, especially in the emerging market, would potentially subsume the effects of other factors on the firms' capital structure (Deesomsak et al., 2004; Sharma \& Paul, 2015). As such, companies with higher concentrated ownership would be more suffered from a higher level of leverage, which means more debt but litter equity. It will serve the purpose 
of strengthening voting power and lowering the possibility of a hostile takeover (Bianco \& Nicodano, 2006; Stulz, 1988). Such problems will be more worsen since the agency conflict and family-owned interest presented a dominant control. Indeed, the profound effect of family ownership toward leverage choices is ample among the family's theory. Primarily GomezMejia, Nunez-Nickel, and Gutierrez (2001), and Gomez-Mejia, Larraza-Kintana, and Makri (2003), characterized the arguments of “the reinforcement the family's power", which means that they may put the high priority on either maintaining the family reputation or reinforcing their power from generation to generation. As a result, family business would preclude external funds and prefer more on internal sources or their owned relationship with a lender. Similar with this argument, Gómez-Mejía, Haynes, Núñez-Nickel, Jacobson, and Moyano-Fuentes (2007) and Sirmon, Arregle, Hitt, and Webb (2008) concerned about "the respondent to the threat", has illustrated that family affiliation with the unique features of socio-emotional, would tend to fasten firm's vision with survival purpose. Consequently, they often decide to maintain longterm preservation and stable wealth creation, leading them to avoid outside funds. Finally, on the view of "agency theory", Berrone, Cruz, \& Gomez-Mejia (2012) decomposed the different idiosyncrasy of family's choices as they responded to risk, verified that family's firm would not be willing to use more equity to react with risk. Instead, they will consider depending on endowment, preservation, or debt to finance the issue.

\section{Data design and regression strategy}

\subsection{Data design}

We obtain data of all listed firms of Vietnam's stock exchange - Ho Chi Minh Stock Exchange (HOSE) from Thomson Reuter Eikon data sources by yearly estimation. We utilize the sample, including 315 companies within five years, from 2013 to 2017 . Noticeably, while most of the important information for corporate finance, ownership structure, and the capital structure was obtained from published reports of Ho Chi Minh Stock Exchange extracting from Thomson Reuter Eikon, the data of family ownership requires more complicated steps. Specifically, we

first access the published reports for each company, which were officially provided by their website. We next hand-checked and cross-checked the board structure and executive information from different company reports, such as annual reports, the prospectus, board of management, corporate governance reports, and shareholders' meeting documents. Finally, filtering the data sources of family and relative members, we categorize and re-code the information to fit with the 6 I P a g e 
variable construction of this study (the detail of variable measurement will be explained right after in this section $)^{5}$

Drawing on Baker and Wurgler (2002) and ElBannan (2017), we construct two separate measurements, namely log of debt and market leverage, to specify the capital structure. The former is estimated by taking a log form of debt volume of a firm (ln_debt). The later proxy is the market leverage, specified by the ratio of book debt divided by the market value of assets (lev_market).

For stock market liquidity, we employ three different indicators of liquidity to strengthen reliability and robustness as follows.

First, following Amihud (2002), the market illiquidity is defined as the weekly ratio of Vietnam's stock return and its weekly trading volume, average over a year:

$$
\mathrm{Illiq}_{i t}=\frac{1}{w_{i t}} \sum_{w=1}^{w_{i t}} \frac{\left|\mathrm{R}_{i w t}\right|}{\mathrm{WVol}_{i w t}}
$$

Where $\left|\mathrm{R}_{i t m}\right|$ is the respective absolute value of the stock return of stock $\mathrm{i}$ on the week w of the year t. $\mathrm{WVol}_{i t m}$ represented for the weekly trading volume in Vietnam Dong, and $\mathrm{w}_{i t}$ illustrates for the number of available weeks for the stock $\mathrm{i}$ in the year $\mathrm{t}$.

Second, Amihud (2002) provided another indicator of market liquidity by looking at share turnover ratio. This ratio is calculated by the weekly trading shares divided by the number of shares traded (in a week) divided by a total number of outstanding shares on that week:

$$
\text { Share_turn }{ }_{i t}=\frac{1}{w_{i t}} \sum_{w=1}^{w_{i t}} \frac{\mathrm{Vol}_{i w t}}{\mathrm{~N}_{i w t}}
$$

Where $\mathrm{VOL}_{i w}$ is the respective weekly trading volume. $\mathrm{N}_{\text {iwt }}$ represents the average number of outstanding shares per week.

Third, following Udomsirikul et al. (2011), and ElBannan (2017), the liquidity is measured by the modified turnover ratio which specified as the weekly number of traded shares divided by the multiplication between a total number of outstanding shares and volatility of earning:

$$
\mathrm{MT}_{i}=\frac{\mathrm{VOL}_{i w}}{\mathrm{~N}_{i w} * \text { Volatility }}
$$

5 The criteria, to construct these family variables, are provided by Gomez-Mejia et al. (2001), Gomez-Mejia et al. (2003), King \& Santor (2008), Setia-Atmaja, Tanewski, \& Skully, 2009, Céspedes et al. (2010), Nguyen (2019). 
Where $\mathrm{VOL}_{i w}$ is the respective weekly trading volume. $\mathrm{N}_{\mathrm{iw}}$ represents the average number of weekly shares outstanding. Volatility is measured by taking the standard deviation of EBIT.

As mentioned above, this study will be utilized by using the data collected from Thomson Reuter Eikon. However, for family variables, motivated by Kien (2020), we use the more complicated process to figure out the family's characteristics in Vietnam context. Indeed, by cross-checking different published reports of the company (firm's prospectus, annual reports, committee reports, and the firm's information on two popular financial websites in Vietnam cafef.vn and vietstock.vn), we provide the three different dichotomous status of family ownership as follows. Family's control will firstly be represented based on the visualization of the family relationship, which is valued by one if there is the presence of family relationships among members of the board of directors (BOD) or the board of managers (BOM), namely fam_con. Secondly, the intensive family's power will be measured by the calculation of a total number of relative members of the origin (origin is the people hold a position in the board of directors), labeled by fam_pow. Noticeably, we only count for the relative members who hold the important positions or own a high proportion of shares. Finally, the level of family's power will be proxied by the voting power of family ownership (estimated by total owning shares of all family members), namely fam_percent.

\subsection{Regression strategy}

Acknowledge the extensive literature from Céspedes et al. (2010); ElBannan (2017); King and Santor (2008); Sharma and Paul (2015), we specify the following model:

$$
\mathrm{Cap}_{i t}=\beta_{0}+\beta_{1} \text { mark_liquid }_{i t}+\beta_{2} \text { fam_own }_{i t}+\beta_{3}(\text { family_own*mark_liquid })_{i t}+\varsigma(\text { control })_{i t}+\varepsilon_{i t}
$$

Where Capit denotes the corporate leverage ratio (or capital structure) of the firm i over the year t. Mar_liqui it is the respective stock market liquidity of the firm $i$ in the year $t$ that has been estimated by three different measurements: market illiquidity (illiq), market share turnover (sh_turn), and market modified turn (mo_turn). Fam_ownit represents for the three different dimensions of family ownership, similar to the above definition, which includes: family control (fam_con), family power (fam_pow), (iii) the voting power of family ownership (fam_percent). Noticeably, fam_own*mark_liquid indicates the interaction between market liquidity and family's control, which presents for the simultaneous effects of both these two variables on firm leverage choice. Meanwhile, Control is the group of control factors that can potentially associate with capital structure choices. Followed ElBannan (2017); Lipson and Mortal (2009); Sharma 
and Paul (2015), we employ the following control variable: firm size, market-to-book ratio (market_book), firm profitability (EBIT to asset ratio), asset tangibility (tangible asset over total asset), non-debt tax shield (the ratio of depreciation over total asset), Vietnam standard industry index (industry) $)^{6}$.

Our model has been applied for the fitted quantile regression for panel data (QRPD), facilitating the mechanism of alternative non-additive fixed-effect (QRPD), non-separable disturbance term, and especially the Markov Chain Monte Carlo framework (Powell, 2016). Canay (2011) suggested that the technique of QRPD can provide the sufficient condition in which the more consistent and asymptotic normal distribution would be identified. Also, the QRPD may generate the pure location shift of parameters, and put to all conditional quantiles under control of exploring heterogeneous covariates estimators rather than the affordable mean effects of the classical fixed and random effects models (Koenker, 2004). Lastly, , Lamarche (2010), and Powell (2016) confirmed the new empirical evidence that QRPD can show the unbiased Gaussian and minimized estimation of asymptotic variance, and jointly estimation which can generally treat for the conditional endogenous problem and non-separable disturbance term.

\section{Empirical results and discussion}

Significantly, our results show that the corporate's capital structure (estimated by the log of debt and market leverage) respond negatively to an increase in market liquidity among three tables of quantile regression (table 1,2,3). Specifically, we discover that the results of most coefficients are robust in three different measures of market liquidity: stock illiquidity - illiq (Amihud, 2002), the share turnover ratio - sh_turn (Amihud, 2002), and the modified turnover ratio - mo_turn (Udomsirikul et al., 2011) ${ }^{7}$. These results are quite similar to the increasing attention of the stream of literature, which finds the negative sign of stock liquidity and capital choice. The higher asymmetric information, the weaker liquid stock would be, leading to lower creditworthiness and less chance of accessing debt markets (ElBannan, 2017). Also, firms with higher stock liquidity can lessen the reliance on the equity cost, and simultaneously strengthen

\footnotetext{
${ }^{6}$ See Table A1 in Appendix for full data description

${ }^{7}$ Besides fitting a quantile regression for panel data (QRPD) developed by Powell (2016), we had made a robust check with the fixed effects model (FEM), and got similar results.
} 
the choice of equity financing, which means a lower level of leverage ratios (Frieder \& Martell, 2006; Hennessy \& Whited, 2005; Kryzanowski et al., 2010; Lipson \& Mortal, 2009).

However, these results are controversial and become positive as the companies are argued to be controlled by the family power. Primarily, as presented in table 1, the interaction of family control (proxied by whether there is the presence of family relationship in the board of directors or the board of managers) with market liquidity: fam_con*illiq; fam_con*sh_turn; and fam_con*mo_turn, may lead to an increase in using more debt since. Similarly, the regression results in table 2 show that the interaction of family power (represented by the total number of relative members in BOD or BOM) with market liquidity (fam_pow*illiq; fam_pow*sh_turn; and fam_pow*mo_turn) have positively impacted on the corporate capital choices. Finally, Table 3 presents the significant increase for the capital structure as the family members increase their voting power (by increasing shares), and the market is more liquid (the interaction of fam_percent*illiq, fam_percent*sh_turn, and fam_percent*mo_turn). These results are entirely consistent with the evidence documented by ElBannan (2017), who finds that stock illiquidity within the issues of information asymmetry and the context of a developing country, can create an adverse selection, leading to lower creditworthiness, to less ability to promote debt finance. Moreover, the above results confirm an exciting conclusion for corporate capital literature since it appears that family power (or also family ownership) - concerning the multiplier mechanism can make the company reluctant to receive more share equity, and prefer more debt (Céspedes et al., 2010; ElBannan, 2017; King \& Santor, 2008; Setia - Atmaja et al., 2009; Sharma \& Paul, 2015). For Vietnam's context, with respect to the link between market liquidity and capital structure, this finding has profoundly differentiated with all previous papers Dang et al. (2019); Moussa and Elgiziry (2019); Thanh and Mai (2017); Tran et al. (2018); Vo (2017) since it confirmed the profound role of family's ownership. 
Table 1. Impacts of the interaction effect of family control and market liquidity on capital structure

\begin{tabular}{|c|c|c|c|c|c|c|c|c|c|}
\hline & quant. 25 & quant. 50 & quant. 75 & quant. 25 & quant. 50 & quant. 75 & quant. 25 & quant. 50 & quant. 75 \\
\hline Depend. Var. 1 & & & & & Indel & & & & \\
\hline illiq & $\begin{array}{r}-47.496 \\
(142.020)\end{array}$ & $\begin{array}{r}414.094 * * * \\
(25.447)\end{array}$ & $\begin{array}{r}469.074 * * * \\
(66.719)\end{array}$ & & & & & & \\
\hline Sh_turn & & & & $\begin{array}{r}-27.890 * * * \\
(1.651)\end{array}$ & $\begin{array}{r}-12.094 * * * \\
(0.408)\end{array}$ & $\begin{array}{r}-14.641 * * * \\
(1.095)\end{array}$ & & & \\
\hline Mo_turn & & & & & & & $\begin{array}{r}-453545.500 * * * \\
(38723.760)\end{array}$ & $\begin{array}{r}-135526.200^{* * *} * \\
(7468.354)\end{array}$ & $\begin{array}{r}-128466.900 * * * \\
(11416.740)\end{array}$ \\
\hline fam_con & $\begin{array}{r}-0.026 * * * \\
(0.009)\end{array}$ & $\begin{array}{r}-0.077 * * * \\
(0.011)\end{array}$ & $\begin{array}{r}-0.056^{* * *} \\
(0.012)\end{array}$ & $\begin{array}{r}-0.125^{* * *} \\
(0.015)\end{array}$ & $\begin{array}{r}-0.075^{* * *} * \\
(0.005)\end{array}$ & $\begin{array}{r}-0.060 * * * \\
(0.010)\end{array}$ & $\begin{array}{r}-0.004 \\
(0.007)\end{array}$ & $\begin{array}{r}-0.017 * * * \\
(0.003)\end{array}$ & $\begin{array}{r}-0.027 * * \\
(0.011)\end{array}$ \\
\hline fam_con*illiq & $\begin{array}{r}-183.962 * * \\
(87.515)\end{array}$ & $\begin{array}{r}-352.140 * * * \\
(27.284)\end{array}$ & $\begin{array}{r}-644.574 * * * \\
(72.204)\end{array}$ & & & & & & \\
\hline fam_con*sh_turn & & & & $\begin{array}{r}21.114 * * * \\
(0.871)\end{array}$ & $\begin{array}{r}8.958 * * * \\
(0.511)\end{array}$ & $\begin{array}{r}8.708 * * * \\
(0.560)\end{array}$ & & & \\
\hline fam_con*mo_turn & & & & & & & $\begin{array}{r}381992.600 * * * \\
(67410.140) \\
\end{array}$ & $\begin{array}{r}24863.540 * * \\
(11455.150) \\
\end{array}$ & $\begin{array}{r}129683.000^{*} \\
(73053.980) \\
\end{array}$ \\
\hline Depend. Var. 2 & \multicolumn{9}{|c|}{ lev_market } \\
\hline illiq & $\begin{array}{r}<0.001 \\
(<0.001)\end{array}$ & $\begin{array}{r}-0.001 \\
(<0.001)\end{array}$ & $\begin{array}{r}0.001 * * * \\
(<0.001)\end{array}$ & & & & & & \\
\hline Sh_turn & & & & $\begin{array}{l}<-0.001 * \\
(<0.001)\end{array}$ & $\begin{array}{r}<-0.001 * * * \\
(<0.001)\end{array}$ & $\begin{array}{r}<-0.001 * * * \\
(<0.001)\end{array}$ & & & \\
\hline Mo_turn & & & & & & & $\begin{array}{r}-0.278^{* * *} \\
(<0.001)\end{array}$ & $\begin{array}{r}-0.199 \\
(<0.001)\end{array}$ & $\begin{array}{r}-0.399 * * * \\
(<0.001)\end{array}$ \\
\hline fam_con & $\begin{array}{r}<-0.001 * * * \\
(<0.001)\end{array}$ & $\begin{array}{r}<-0.001 * * * \\
(<0.001)\end{array}$ & $\begin{array}{r}<-0.001 * * * \\
(<0.001)\end{array}$ & $\begin{array}{r}<-0.001 * * * \\
(<0.001)\end{array}$ & $\begin{array}{r}<-0.001 * * * \\
(<0.001)\end{array}$ & $\begin{array}{r}<-0.001 * * * \\
(<0.001)\end{array}$ & $\begin{array}{l}<-0.001 \\
(<0.001)\end{array}$ & $\begin{array}{l}<-0.001 \\
(<0.001)\end{array}$ & $\begin{array}{r}<-0.001 * * * \\
(<0.001)\end{array}$ \\
\hline fam_con*illiq & $\begin{array}{l}<-0.001 \\
(<0.001)\end{array}$ & $\begin{array}{l}<-0.001^{*} \\
(<0.001)\end{array}$ & $\begin{array}{r}<-0.001 * * \\
(<0.001)\end{array}$ & & & & & & \\
\hline fam_con*sh_turn & & & & $\begin{array}{r}<0.001 * * * \\
(<0.001)\end{array}$ & $\begin{array}{r}<0.001 * * * \\
(<0.001)\end{array}$ & $\begin{array}{r}<0.001 \\
(<0.001)\end{array}$ & & & \\
\hline fam_con*mo_turn & & & & & & & $\begin{array}{r}0.026 \\
(0.117)\end{array}$ & $\begin{array}{l}0.344^{*} \\
(0.191)\end{array}$ & $\begin{array}{r}0.342 * * * \\
(0.105)\end{array}$ \\
\hline No. Obs. & 1198 & 1198 & 1198 & 1198 & 1198 & 1198 & 1198 & 1198 & 1198 \\
\hline
\end{tabular}

11 P a g e 
Table 2. Impacts of the interaction effect of family’s power and market liquidity on capital structure

\begin{tabular}{|c|c|c|c|c|c|c|c|c|c|}
\hline & \multicolumn{9}{|c|}{ lev_Indebt } \\
\hline Illiq & $\begin{array}{r}\text { quant. } 25 \\
364.220 * * * \\
(78.133)\end{array}$ & $\begin{array}{r}\text { quant. } 50 \\
46.738 \\
(84.874)\end{array}$ & $\begin{array}{r}\text { quant. 75 } \\
65.480 \\
(203.167)\end{array}$ & quant. 25 & quant. $\overline{50}$ & quant. 75 & quant. 25 & quant. 50 & quant. 75 \\
\hline Sh_turn & & & & $\begin{array}{r}-26.942 * * * \\
(2.171)\end{array}$ & $\begin{array}{r}-8.997 * * * \\
(1.468)\end{array}$ & $\begin{array}{r}-6.879 * * * \\
(0.398)\end{array}$ & & & \\
\hline Mo_turn & & & & & & & $\begin{array}{r}-647458.900 * * * \\
(59976.920)\end{array}$ & $\begin{array}{r}-237837.000 * * * \\
(26920.100)\end{array}$ & $\begin{array}{r}-169339.700 * * * \\
(24468.800)\end{array}$ \\
\hline Fam_pow & $\begin{array}{r}-0.008 * * * \\
(<0.001)\end{array}$ & $\begin{array}{r}-0.012 * * * \\
(<0.001)\end{array}$ & $\begin{array}{r}-0.007 * * * \\
(<0.001)\end{array}$ & $\begin{array}{r}-0.012 * * * \\
(0.002)\end{array}$ & $\begin{array}{r}-0.004 * * * \\
(<0.001)\end{array}$ & $\begin{array}{r}-0.003^{* * *} \\
(<0.001)\end{array}$ & $\begin{array}{r}-0.010^{* * *} \\
(<0.001)\end{array}$ & $\begin{array}{r}-0.006^{* * *} \\
(<0.001)\end{array}$ & $\begin{array}{l}-0.002 * * \\
(<0.001)\end{array}$ \\
\hline Fam_pow*illiq & $\begin{array}{r}-63.641^{* *} \\
(27.902)\end{array}$ & $\begin{array}{c}-44.792^{*} \\
(23.253)\end{array}$ & $\begin{array}{r}-173.788 * * * \\
(49.735)\end{array}$ & & & & & & \\
\hline Fam_pow*sh_turn & & & & $\begin{array}{r}3.760 * * * \\
(0.570)\end{array}$ & $\begin{array}{r}2.425 * * * \\
(0.580)\end{array}$ & $\begin{array}{r}1.522 * * * \\
(0.165)\end{array}$ & & & \\
\hline \multirow[t]{2}{*}{ fam_pow*mo_turn } & & & & & & & $\begin{array}{r}103628.200 * * * \\
(7157.584)\end{array}$ & $\begin{array}{r}72901.550 * * * \\
(10880.650) \\
\end{array}$ & $\begin{array}{r}26467.530 * * * * \\
(4963.908) \\
\end{array}$ \\
\hline & \multicolumn{9}{|c|}{ lev_market } \\
\hline Illiq & $\begin{array}{r}<0.001 * * \\
(<0.001)\end{array}$ & $\begin{array}{r}<0.001 \\
(<0.001)\end{array}$ & $\begin{array}{r}<0.001 * * \\
(<0.001)\end{array}$ & & & & & & \\
\hline Sh_turn & & & & $\begin{array}{r}<-0.001 * * * \\
(<0.001)\end{array}$ & $\begin{array}{r}<-0.001 * * * \\
(<0.001)\end{array}$ & $\begin{array}{r}<-0.001^{* * * *} \\
(<0.001)\end{array}$ & & & \\
\hline Mo_turn & & & & & & & $\begin{array}{r}-0.288^{* *} \\
(0.129)\end{array}$ & $\begin{array}{r}-0.561 * * * \\
(0.163)\end{array}$ & $\begin{array}{r}-0.905 * * * \\
(0.137)\end{array}$ \\
\hline Fam_pow & $\begin{array}{r}<-0.001 * * * \\
(<0.001)\end{array}$ & $\begin{array}{r}<-0.001 * * * \\
(<0.001)\end{array}$ & $\begin{array}{r}<-0.001 * * * \\
(<0.001)\end{array}$ & $\begin{array}{r}<-0.001^{* * * *} \\
(<0.001)\end{array}$ & $\begin{array}{r}<-0.001 * * * \\
(<0.001)\end{array}$ & $\begin{array}{r}<-0.001^{* * * *} \\
(<0.001)\end{array}$ & $\begin{array}{r}<-0.001 * * * \\
(<0.001)\end{array}$ & $\begin{array}{r}<-0.001 * * * \\
(<0.001)\end{array}$ & $\begin{array}{r}<-0.001 * * * \\
(<0.001)\end{array}$ \\
\hline Fam_pow*illiq & $\begin{array}{r}<-0.001 * * \\
(<0.001)\end{array}$ & $\begin{array}{r}<-0.001 \\
(<0.001)\end{array}$ & $\begin{array}{r}<-0.001 \\
(<0.001)\end{array}$ & & & & & & \\
\hline Fam_pow*sh_turn & & & & $\begin{array}{r}<0.001 * * * \\
(<0.001)\end{array}$ & $\begin{array}{r}<0.001 * * * \\
(<0.001)\end{array}$ & $\begin{array}{r}<0.001 * * * \\
(<0.001)\end{array}$ & & & \\
\hline Fam_pow*mo_turn & & & & & & & $\begin{array}{r}0.246^{* * *} \\
(0.009)\end{array}$ & $\begin{array}{r}0.440 * * * \\
(0.104)\end{array}$ & $\begin{array}{r}0.688 * * * \\
(0.065)\end{array}$ \\
\hline No. Obs. & 1198 & 1198 & 1198 & 1198 & 1198 & 1198 & 1198 & 1198 & 1198 \\
\hline
\end{tabular}

12| P a g e 
Table 3. Impacts of the interaction effect of the voting power of family ownership and market liquidity on capital structure

\begin{tabular}{|c|c|c|c|c|c|c|c|c|c|}
\hline & \multicolumn{9}{|c|}{ lev_Indebt } \\
\hline & quant. 25 & quant. 50 & quant. 75 & quant. 25 & quant. 50 & quant. 75 & quant. 25 & quant. 50 & quant. 75 \\
\hline illiq & $\begin{array}{r}185.938 * * * \\
(48.209)\end{array}$ & $\begin{array}{r}391.476 * * * \\
(150.388)\end{array}$ & $\begin{array}{r}464.513 * * * \\
(108.176)\end{array}$ & & & & & & \\
\hline Sh_turn & & & & $\begin{array}{r}1.330 \\
(2.787)\end{array}$ & $\begin{array}{r}-489236.100 * * * \\
(49067.090)\end{array}$ & $\begin{array}{r}-6.517 * * \\
(2.578)\end{array}$ & & & \\
\hline Mo_turn & & & & & & & $\begin{array}{r}-489236.100 * * * \\
(49067.090)\end{array}$ & $\begin{array}{r}-124508.200^{* * * *} \\
(9538.592)\end{array}$ & $\begin{array}{r}-31340.620^{* *} \\
(13855.170)\end{array}$ \\
\hline fam_percent & $\begin{array}{r}-0.091 * * \\
(0.045)\end{array}$ & $\begin{array}{r}0.078 \\
(0.081)\end{array}$ & $\begin{array}{r}0.024 \\
(0.056)\end{array}$ & $\begin{array}{r}-0.450 * * * \\
(0.079)\end{array}$ & $\begin{array}{l}-0.060 \\
(0.094)\end{array}$ & $\begin{array}{l}-0.017 \\
(0.028)\end{array}$ & $\begin{array}{r}-0.060 \\
(0.094)\end{array}$ & $\begin{array}{r}-0.156^{* * *} \\
(0.015)\end{array}$ & $\begin{array}{r}-0.245 * * * \\
(0.031)\end{array}$ \\
\hline Fam_percent*illiq & $\begin{array}{r}-1063.000 \\
(688.557)\end{array}$ & $\begin{array}{r}-3611.903 * * * \\
(1079.296)\end{array}$ & $\begin{array}{r}-4738.679 * * * \\
(1831.787)\end{array}$ & & & & & & \\
\hline Fam_percent*sh_turn & & & & $\begin{array}{r}62.131 * * * \\
(5.494)\end{array}$ & $\begin{array}{r}79167.78 \\
(116323.500)\end{array}$ & $\begin{array}{r}27.151 \\
(21.671)\end{array}$ & & & \\
\hline \multirow[t]{2}{*}{ Fam_percent*mo_turn } & & & & & & & $\begin{array}{r}79167.780 \\
(116323.500) \\
\end{array}$ & $\begin{array}{r}914429.800 * * * \\
(17280.020) \\
\end{array}$ & $\begin{array}{r}1057921.000 * * * \\
(60616.990) \\
\end{array}$ \\
\hline & \multicolumn{9}{|c|}{ lev_market } \\
\hline illiq & $\begin{array}{l}<0.001^{* *} \\
(<0.001)\end{array}$ & $\begin{array}{l}<0.001^{* *} \\
(<0.001)\end{array}$ & $\begin{array}{r}<0.001 * * \\
(<0.001)\end{array}$ & & & & & & \\
\hline Sh_turn & & & & $\begin{array}{r}<-0.001 * * * \\
(<0.001)\end{array}$ & $\begin{array}{r}<0.001 \\
(<0.001)\end{array}$ & $\begin{array}{r}<-0.001 * * * \\
\quad(<0.001)\end{array}$ & & & \\
\hline Mo_turn & & & & & & & $\begin{array}{l}-0.016 \\
(0.050)\end{array}$ & $\begin{array}{r}-0.333^{* * *} \\
(0.118)\end{array}$ & $\begin{array}{r}-0.320 \\
(0.312)\end{array}$ \\
\hline fam_percent & $\begin{array}{r}<-0.001 * * * \\
(<0.001)\end{array}$ & $\begin{array}{l}<-0.001 \\
(<0.001)\end{array}$ & $\begin{array}{r}<-0.001 * * * \\
(<0.001)\end{array}$ & $\begin{array}{l}<-0.001 \\
(<0.001)\end{array}$ & $\begin{array}{l}<-0.001 * \\
(<0.001)\end{array}$ & $\begin{array}{r}<-0.001 * * \\
(<0.001)\end{array}$ & $\begin{array}{r}<-0.001 * * \\
(<0.001)\end{array}$ & $\begin{array}{r}<-0.001 \\
(<0.001)\end{array}$ & $\begin{array}{l}<-0.001 * \\
(<0.001)\end{array}$ \\
\hline fam_percent*illiq & $\begin{array}{r}-0.002 * * \\
(0.001)\end{array}$ & $\begin{array}{r}<-0.001 \\
(0.001)\end{array}$ & $\begin{array}{r}-0.007 * * * \\
(0.002)\end{array}$ & & & & & & \\
\hline Fam_percent*sh_turn & & & & $\begin{array}{r}<0.001 \\
(<0.001)\end{array}$ & $\begin{array}{r}<0.001 * * * \\
(<0.001)\end{array}$ & $\begin{array}{r}<0.001 \\
(<0.001)\end{array}$ & & & \\
\hline Fam_percent*mo_turn & & & & & & & $\begin{array}{r}0.182 \\
(0.193)\end{array}$ & $\begin{array}{r}0.759 \\
(0.719)\end{array}$ & $\begin{array}{l}0.637 * \\
(0.376)\end{array}$ \\
\hline No. Obs. & 1198 & 1198 & 1198 & 1198 & 1198 & 1198 & 1198 & 1198 & 1198 \\
\hline
\end{tabular}

Notes: The table shows the panel quantile regression for causal relationship between market liquidity (respected by three different dimensions of illiquidity, share turnover ratio, and modified turnover) and corporate leverage (two proxies of log of debt and market leverage) as the company is argued to be controlled by the voting power of family ownership (measured by total number of owning shares of all family members). *** denotes the significance at the $1 \%$, ** $5 \%$, and $* 10 \%$ level, respectively with t-statistic in the parenthesis. See Table A1 in Appendix for the full estimation results with explicit estimations of the control variables. 


\section{Conclusion}

Using a new prominent case of the ASEAN region, namely Vietnam, of 315 listed firms during five years sample (2013-2017), this study documents the four critical contributions to the literature. Firstly, there is a negative impact of market liquidity on corporate leverage (for both three cases of liquidity and two measurements of leverage). This result is profoundly valuable for the new prominent case of the ASEAN region, namely Vietnam, rather than the developed market sample (Kryzanowski et al., 2010; Sharma \& Paul, 2015), or some other areas such as Thailand (Udomsirikul et al., 2011), and Egypt (ElBannan, 2017). Second, this study can be one of the first of its kind in current literature when it demonstrates the mediation effect of family ownership on the relationship between market liquidity and capital structure. It is worth noting that family ownership with the dual-class control may have an adverse effect that tends to employ more control-enhancement than the non-family firm, leading to a higher level of risk aversion for debt-bankruptcy. Third, this study has provided new empirical evidence for raising family ownership in Vietnam's context. This contribution, exclusively for the capital structure, is totally significant and profoundly different from all previous studies of determinants of capital structure in the Vietnam market (Dang et al., 2019; Moussa \& Elgiziry, 2019); D. Nguyen et al. (2012); (Thanh \& Mai, 2017; Tran et al., 2018; X. V. Vo, 2017).

Our results shed light on the current literature and current practical market operation that may have implications. Firstly, this study may raise a satisfying conclusion for financial managers and planners. As there is higher asymmetric information or weak liquid stock, it would lead the firm to lower creditworthiness, making the firm have less chance of accessing debt markets. In this manner, the administration has to reconsider and readjust their situation to improve their stock liquidity. Secondly, based on these results, the authority, mainly in the Vietnam market, should strengthen their problem of asymmetric information that would foster more chances for the market to access different debt financing sources. Last but not least, the results of this research would try the best of author's knowledge, to call for the highlight interests of family ownership in new emerging countries where there is lack of good corporate governance quality, then can help to facilitate more research for finance decision and family's intervention. 


\section{Appendix A}

Table A1. Data description

\begin{tabular}{lrrrrr}
\hline Variable & Obs & Mean & Std. Dev. & Min & Max \\
\hline Total debt & 1,548 & $2.26 \mathrm{E}+06$ & $7.84 \mathrm{E}+06$ & $9.36 \mathrm{E}+00$ & $1.61 \mathrm{E}+08$ \\
Market capitalization & 1,384 & $3.47 \mathrm{E}+12$ & $1.53 \mathrm{E}+13$ & $6.55 \mathrm{E}+09$ & $2.20 \mathrm{E}+14$ \\
Total asset & 1,548 & $1.81 \mathrm{E}+07$ & $5.55 \mathrm{E}+08$ & $9.97 \mathrm{E}+02$ & $2.18 \mathrm{E}+10$ \\
Lndebt & 1,548 & 13.197 & 1.704 & 2.236 & 18.898 \\
Lev_market & 1,384 & $1.93 \mathrm{E}-06$ & $3.03 \mathrm{E}-06$ & $2.27 \mathrm{E}-09$ & $4.59 \mathrm{E}-05$ \\
Fam_con & 1,561 & 0.471 & 0.499 & 0.000 & 1.000 \\
Fam_pow & 1,561 & 2.744 & 6.198 & 0.000 & 100.000 \\
Family_percent & 1,561 & 0.048 & 0.124 & 0.000 & 1.080 \\
Mark_illiquid & 1,379 & $7.00 \mathrm{E}-06$ & $9.16 \mathrm{E}-05$ & $-8.94 \mathrm{E}-04$ & $1.09 \mathrm{E}-03$ \\
Mark_shareturn & 1,379 & $3.79 \mathrm{E}-03$ & $6.12 \mathrm{E}-03$ & $3.29 \mathrm{E}-06$ & $5.69 \mathrm{E}-02$ \\
Mark_moditurn & 1,379 & $1.11 \mathrm{E}-07$ & $3.43 \mathrm{E}-07$ & $2.62 \mathrm{E}-11$ & $7.87 \mathrm{E}-06$ \\
Profit & 1,543 & 0.095 & 0.105 & -1.644 & 0.997 \\
Tang_ass & 1,548 & 0.196 & 0.209 & 0.000 & 0.940 \\
Depre_ass & 1,544 & -0.200 & 0.232 & -1.742 & 0.000 \\
\hline
\end{tabular}


Table A2. Variable description

\begin{tabular}{|c|c|}
\hline Variables & Definitions \\
\hline \multicolumn{2}{|c|}{ Corporate capital structure(or leverage) } \\
\hline $\operatorname{Ln}(\text { debt })_{i, t}$ & The log form of debt volume by each firm in each year. \\
\hline Lev_market,$t_{1}$ & $\begin{array}{l}\text { The market leverage measured by the ratio of book debt divided by the market value of } \\
\text { assets by each firm each year. Noticeably, the market value of an asset will be measured } \\
\text { by subtracting the total asset to total equity, then adding market equity (where market } \\
\text { equity is calculated by the multiplication of the outstanding shares to the share price) }\end{array}$ \\
\hline \multicolumn{2}{|c|}{ Stock market liquidity } \\
\hline Illiq $_{\mathrm{i}, \mathrm{t}}$ & $\begin{array}{l}\text { the stock illiquidity was estimated by Amihud (2002), reveals the weekly stock return } \\
\text { as a percentage of its volume trading on the specific Vietnamese unit, average over a } \\
\text { year: } \\
\qquad \mathrm{Illiq}_{i t}=\frac{1}{\mathrm{~m}_{i t}} \sum_{m=1}^{m_{i t}} \frac{\left|\mathrm{R}_{i m t}\right|}{\mathrm{MVol}_{i m t}}\end{array}$ \\
\hline Sh_turn $_{i, t}$ & $\begin{array}{l}\text { The share turnover ratio. This ratio is calculated by the weekly trading shares divided } \\
\text { by a total number of outstanding shares on that week: } \\
\qquad \text { Share_turn }{ }_{i t}=\frac{1}{m_{i t}} \sum_{m=1}^{m_{i t}} \frac{\mathrm{Vol}_{i m t}}{\mathrm{~N}_{i m t}}\end{array}$ \\
\hline Mo_turn & $\begin{array}{l}\text { The liquidity is measured by the turnover ratio, which specified as the weekly number } \\
\text { of traded shares divided by the multiplication between the total number of outstanding } \\
\text { shares and volatility of earning: } \\
\qquad \mathrm{MT}_{i}=\frac{\mathrm{VOL}_{\text {im }}}{\mathrm{N}_{i m} * \text { Volatility }}\end{array}$ \\
\hline \multicolumn{2}{|l|}{ Family control } \\
\hline Fam_con & $\begin{array}{l}\text { The family power is defined by the presence of a family relationship in the board of } \\
\text { directors (BOD) or of managers (BOM) }\end{array}$ \\
\hline Fam_pow & $\begin{array}{l}\text { The level of family's attendance - the number of people who hold the important } \\
\text { positions or obtain the high proportion of shares, have a relative relationship with the } \\
\text { directors }\end{array}$ \\
\hline Fam_percent & $\begin{array}{l}\text { The level of family's owning shares measured by the total number of owning shares of } \\
\text { all family member }\end{array}$ \\
\hline \multicolumn{2}{|c|}{ Financial control } \\
\hline Firm_size & Log of total asset \\
\hline Market_book & $\begin{array}{l}\text { The market to book ratio is calculated as the market capitalization divided by net book } \\
\text { value. Where net book value equals total asset minus total liability }\end{array}$ \\
\hline Profit & Firm's profitability = EBIT/ total asset \\
\hline Tang_ass & The asset tangibility/ total asset \\
\hline Depre_ass & The non-debt tax shield is proxied for the ratios of depreciation to total asset \\
\hline Industry & $\begin{array}{l}\text { The Vietnam standard industry codes. It is used for } \\
\text { controlling the characteristics of the industry }\end{array}$ \\
\hline
\end{tabular}




\section{Appendix B}

Table B1.1. Estimation Results of Panel quantile regression with Estimated Coefficients of all Control Variables. The case of ln debt and family control.

\begin{tabular}{|c|c|c|c|c|c|c|c|c|c|}
\hline & \multicolumn{9}{|c|}{ Ln_debt } \\
\hline & quant. 25 & quant. 50 & quant. 75 & quant. 25 & quant. 50 & quant. 75 & quant. 25 & quant. 50 & quant. 75 \\
\hline Illiq & $\begin{array}{r}-47.496 \\
(142.020)\end{array}$ & $\begin{array}{r}414.094 * * * \\
(25.447)\end{array}$ & $\begin{array}{r}469.074 * * * \\
(66.719)\end{array}$ & & & & & & \\
\hline Sh_turn & & & & $\begin{array}{r}-27.890 * * * \\
(1.651)\end{array}$ & $\begin{array}{r}-12.094 * * * \\
(0.408)\end{array}$ & $\begin{array}{r}-14.641 * * * \\
(1.095)\end{array}$ & & & \\
\hline Mo_turn & & & & & & & $\begin{array}{r}-453545.5^{* * *} \\
(38723.76)\end{array}$ & $\begin{array}{r}-135526.2 * * * \\
(7468.354)\end{array}$ & $\begin{array}{r}-128466.9 * * * \\
(11416.74)\end{array}$ \\
\hline Fam_con & $\begin{array}{r}-0.026^{* * *} \\
(0.009)\end{array}$ & $\begin{array}{r}-0.077 * * * \\
(0.011)\end{array}$ & $\begin{array}{r}-0.056^{* * *} \\
(0.012)\end{array}$ & $\begin{array}{r}-0.125 * * * \\
(0.015)\end{array}$ & $\begin{array}{r}-0.075^{* * * *} \\
(0.005)\end{array}$ & $\begin{array}{r}-0.060 * * * \\
(0.010)\end{array}$ & $\begin{array}{r}-0.004 \\
(0.007)\end{array}$ & $\begin{array}{r}-0.017^{* * * *} \\
(0.003)\end{array}$ & $\begin{array}{r}-0.027^{* *} \\
(0.011)\end{array}$ \\
\hline $\begin{array}{l}\text { Fam_con* } \\
\text { illiq }\end{array}$ & $\begin{array}{r}-183.962 * * \\
(87.515)\end{array}$ & $\begin{array}{r}-352.14 * * * \\
(27.284)\end{array}$ & $\begin{array}{r}-644.574 * * * \\
(72.204)\end{array}$ & & & & & & \\
\hline $\begin{array}{l}\text { Fam_con* } \\
\text { sh_turn }\end{array}$ & & & & $\begin{array}{r}21.114 * * * \\
(0.871)\end{array}$ & $\begin{array}{r}8.958 * * * \\
(0.511)\end{array}$ & $\begin{array}{r}8.708^{* * *} \\
(0.560)\end{array}$ & & & \\
\hline $\begin{array}{l}\text { Fam_con* } \\
\text { mo_turn }\end{array}$ & & & & & & & $\begin{array}{r}381992.6 * * * \\
(67410.14)\end{array}$ & $\begin{array}{r}24863.54^{* *} \\
(11455.15)\end{array}$ & $\begin{array}{r}129683.000 * \\
(73053.98)\end{array}$ \\
\hline Firm_size & $\begin{array}{r}1.165^{* * *} \\
(0.007)\end{array}$ & $\begin{array}{r}1.167 * * * \\
(0.006)\end{array}$ & $\begin{array}{r}1.178^{* * * *} \\
(0.005)\end{array}$ & $\begin{array}{r}1.167 * * * \\
(0.004)\end{array}$ & $\begin{array}{r}1.089^{* * * *} \\
(0.002)\end{array}$ & $\begin{array}{r}1.061^{* * * *} \\
(0.005)\end{array}$ & $\begin{array}{r}1.136^{* * * *} \\
(0.004)\end{array}$ & $\begin{array}{r}1.110^{* * *} \\
(0.002)\end{array}$ & $\begin{array}{r}1.100 * * * \\
(0.011)\end{array}$ \\
\hline Market_book & $\begin{array}{r}-0.000 * * * \\
(0.000)\end{array}$ & $\begin{array}{r}-0.000 \\
(0.000)\end{array}$ & $\begin{array}{r}-0.000 * * * \\
(0.000)\end{array}$ & $\begin{array}{r}-0.000 \\
(0.000)\end{array}$ & $\begin{array}{r}-0.000 * * * \\
(0.000)\end{array}$ & $\begin{array}{r}0.000^{* * * *} \\
(0.000)\end{array}$ & $\begin{array}{r}0.000^{* * * *} \\
(0.000)\end{array}$ & $\begin{array}{r}-0.000 * * * \\
(0.000)\end{array}$ & $\begin{array}{r}-0.000^{* *} \\
(0.000)\end{array}$ \\
\hline Profit & $\begin{array}{r}-1.737 * * * \\
(0.076)\end{array}$ & $\begin{array}{r}-2.048^{* * *} \\
(0.051)\end{array}$ & $\begin{array}{r}-1.537 * * * \\
(0.082)\end{array}$ & $\begin{array}{r}-2.004 * * * \\
(0.059)\end{array}$ & $\begin{array}{r}-1.922 * * * \\
(0.072)\end{array}$ & $\begin{array}{r}-1.355^{* * *} \\
(0.219)\end{array}$ & $\begin{array}{r}-2.632 * * * \\
(0.052)\end{array}$ & $\begin{array}{r}-2.500 * * * \\
(0.029)\end{array}$ & $\begin{array}{r}-2.162 * * * \\
(0.041)\end{array}$ \\
\hline Tang_ass & $\begin{array}{r}0.454 * * * \\
(0.023)\end{array}$ & $\begin{array}{r}0.312 * * * \\
(0.022)\end{array}$ & $\begin{array}{r}0.434 * * * \\
(0.020)\end{array}$ & $\begin{array}{r}0.373 * * * \\
(0.037)\end{array}$ & $\begin{array}{r}0.057 * * \\
(0.025)\end{array}$ & $\begin{array}{r}0.107^{* * *} \\
(0.015)\end{array}$ & $\begin{array}{r}0.618^{* * *} \\
(0.029)\end{array}$ & $\begin{array}{r}0.061^{* * * *} \\
(0.009)\end{array}$ & $\begin{array}{r}0.000 \\
(0.050)\end{array}$ \\
\hline Depre_ass & $\begin{array}{r}0.589 * * * \\
(0.033)\end{array}$ & $\begin{array}{r}0.513 * * * \\
(0.011)\end{array}$ & $\begin{array}{r}0.418^{* * * *} \\
(0.025)\end{array}$ & $\begin{array}{r}0.565^{* * * *} \\
(0.036)\end{array}$ & $\begin{array}{r}0.293 * * * \\
(0.031)\end{array}$ & $\begin{array}{r}0.383 * * * \\
(0.022)\end{array}$ & $\begin{array}{r}0.623 * * * \\
(0.031)\end{array}$ & $\begin{array}{r}0.234 * * * \\
(0.006)\end{array}$ & $\begin{array}{r}0.302 * * * \\
(0.063)\end{array}$ \\
\hline Industry & $\begin{array}{r}0.008^{* * *} \\
(0.002)\end{array}$ & $\begin{array}{r}0.009 * * * \\
(0.001)\end{array}$ & $\begin{array}{r}-0.005^{* *} \\
(0.002)\end{array}$ & $\begin{array}{r}0.004 \\
(0.002)\end{array}$ & $\begin{array}{r}0.011^{* * * *} \\
(0.002)\end{array}$ & $\begin{array}{r}-0.003 \\
(0.005)\end{array}$ & $\begin{array}{r}0.050 * * * \\
(0.003)\end{array}$ & $\begin{array}{r}0.015^{* * *} \\
(0.001)\end{array}$ & $\begin{array}{r}0.017^{* * *} * \\
(0.003)\end{array}$ \\
\hline No. Obs. & 1198 & 1198 & 1198 & 1198 & 1198 & 1198 & 1198 & 1198 & 1198 \\
\hline
\end{tabular}


Table B1.2. Estimation Results of Panel quantile regression with Estimated Coefficients of all Control Variables. The case of market leverage and family control.

\begin{tabular}{|c|c|c|c|c|c|c|c|c|c|}
\hline & \multicolumn{9}{|c|}{ lev_market } \\
\hline & quant. 25 & quant. 50 & quant. 75 & quant. 25 & quant. 50 & quant. 75 & quant. 25 & quant. 50 & quant. 75 \\
\hline Illiq & $\begin{array}{r}0.0000148 \\
(0.0000827)\end{array}$ & $\begin{array}{r}-0.0001637 \\
(0.0001447)\end{array}$ & $\begin{array}{r}0.0008081^{* * * *} \\
(0.0002322)\end{array}$ & & & & & & \\
\hline Sh_turn & & & & $\begin{array}{l}-0.00000257^{*} \\
(0.00000137)\end{array}$ & $\begin{array}{r}-0.000015^{* * *} \\
(0.00000163)\end{array}$ & $\begin{array}{r}-0.0000352 * * * \\
(0.00000628)\end{array}$ & & & \\
\hline Mo_turn & & & & & & & $\begin{array}{r}-0.277505 * * * \\
(0.1067487)\end{array}$ & $\begin{array}{c}-0.1992705 \\
(0.1398426)\end{array}$ & $\begin{array}{r}-0.3986924 * * * \\
(0.1476881)\end{array}$ \\
\hline Fam_con & $\begin{array}{r}-0.000000035^{* * * *} \\
(0.000000009)\end{array}$ & $\begin{array}{r}-0.000000090 * * * \\
(0.000000011)\end{array}$ & $\begin{array}{r}-0.000000111 * * * \\
(0.000000018)\end{array}$ & $\begin{array}{r}-0.000000101 * * * \\
(0.000000020)\end{array}$ & $\begin{array}{r}-0.000000196 * * * \\
(0.000000007)\end{array}$ & $\begin{array}{r}-0.000000592 * * * \\
(0.000000072)\end{array}$ & $\begin{array}{r}-0.000000052 \\
(0.000000033)\end{array}$ & $\begin{array}{r}-0.000000041 \\
(0.000000096)\end{array}$ & $\begin{array}{r}-0.000000225^{* * *} * \\
(0.000000054)\end{array}$ \\
\hline $\begin{array}{l}\text { Fam_con* } \\
\text { illiq }\end{array}$ & $\begin{array}{r}-0.0000822 \\
(0.0000584)\end{array}$ & $\begin{array}{r}-0.0003375^{*} \\
(0.000196)\end{array}$ & $\begin{array}{r}-0.0011364^{* *} \\
(0.0005299)\end{array}$ & & & & & & \\
\hline $\begin{array}{l}\text { Fam_con* } \\
\text { sh_turn }\end{array}$ & & & & $\begin{array}{r}0.0000299 * * * \\
(0.00000232)\end{array}$ & $\begin{array}{r}0.00002 * * * \\
(0.00000222)\end{array}$ & $\begin{array}{r}0.00000146 \\
(0.00000767)\end{array}$ & & & \\
\hline $\begin{array}{l}\text { Fam_con* } \\
\text { mo_turn }\end{array}$ & & & & & & & $\begin{array}{r}0.0257907 \\
(0.1166009)\end{array}$ & $\begin{array}{c}0.3435067^{*} \\
(0.1919383)\end{array}$ & $\begin{array}{r}0.3419755^{* * *} \\
(0.1051939)\end{array}$ \\
\hline Firm_size & $\begin{array}{r}0.000000134 * * * \\
(0.000000003)\end{array}$ & $\begin{array}{r}0.000000164 * * * \\
(0.000000015)\end{array}$ & $\begin{array}{r}0.000000186^{* * *} \\
(0.000000020)\end{array}$ & $\begin{array}{r}0.000000117^{* * *} \\
(0.000000004)\end{array}$ & $\begin{array}{r}0.000000127 * * * \\
(0.000000006)\end{array}$ & $\begin{array}{r}0.0000003 * * * \\
(0.000000037)\end{array}$ & $\begin{array}{r}0.000000142 * * * \\
(0.000000042)\end{array}$ & $\begin{array}{r}0.000000168 * * * \\
(0.000000048)\end{array}$ & $\begin{array}{r}0.000000198 * * * \\
(0.000000015)\end{array}$ \\
\hline Market_book & $\begin{array}{r}-0.000000000^{* * *} \\
(0.000000000)\end{array}$ & $\begin{array}{r}-0.000000000^{* * * *} \\
(0.000000000)\end{array}$ & $\begin{array}{r}-0.000000000^{* * *}(0.000000000)\end{array}$ & $\begin{array}{r}-0.000000000 * * * \\
(0.000000000)\end{array}$ & $\begin{array}{r}-0.000000000^{* * * *} \\
(0.000000000)\end{array}$ & $\begin{array}{r}-0.000000000 * * * \\
(0.000000000)\end{array}$ & $\begin{array}{r}-0.000000000^{* * * *} \\
(0.000000000)\end{array}$ & $\begin{array}{r}-0.000000000 * * * \\
(0.000000000)\end{array}$ & $\begin{array}{r}-0.000000000 * * * \\
(0.000000000)\end{array}$ \\
\hline Profit & $\begin{array}{r}-0.00000117 * * * \\
(0.000000047)\end{array}$ & $\begin{array}{r}-0.00000407^{* * * *} \\
(0.000000101)\end{array}$ & $\begin{array}{r}-0.00000429^{* * *} \\
(0.000000059)\end{array}$ & $\begin{array}{r}-0.000000959 * * * \\
(0.000000079)\end{array}$ & $\begin{array}{r}-0.00000426^{* * * *} \\
(0.000000105)\end{array}$ & $\begin{array}{r}-0.00000594 * * * \\
(0.00000018)\end{array}$ & $\begin{array}{r}-0.00000356 * * * \\
(0.000000371)\end{array}$ & $\begin{array}{r}-0.0000038^{* * * *} \\
(0.000000331)\end{array}$ & $\begin{array}{r}-0.0000059^{* * * *} \\
(0.000000461)\end{array}$ \\
\hline Tang_ass & $\begin{array}{r}-0.000000218^{* * * *} \\
(0.000000027)\end{array}$ & $\begin{array}{r}-0.000000173 * * * \\
(0.000000054)\end{array}$ & $\begin{array}{r}0.000000005 \\
(0.000000066)\end{array}$ & $\begin{array}{r}-0.000000027 \\
(0.000000025)\end{array}$ & $\begin{array}{r}-0.000000133^{* * *} \\
(0.000000021)\end{array}$ & $\begin{array}{r}-0.000000754^{*} \\
(0.000000398)\end{array}$ & $\begin{array}{r}-0.000000365^{* *} \\
(0.000000166)\end{array}$ & $\begin{array}{r}-0.000000021 \\
(0.000000058)\end{array}$ & $\begin{array}{r}-0.000000426^{* * * *} \\
(0.000000112)\end{array}$ \\
\hline Depre_ass & $\begin{array}{r}0.000000165^{* * * *} \\
(0.000000020)\end{array}$ & $\begin{array}{r}0.000000525^{* * *} \\
(0.000000037)\end{array}$ & $\begin{array}{r}0.000000575^{* * * *} \\
(0.000000045)\end{array}$ & $\begin{array}{r}0.000000363^{* * *} \\
(0.000000026)\end{array}$ & $\begin{array}{r}0.00000053^{* * *} \\
(0.000000025)\end{array}$ & $\begin{array}{r}0.00000126^{* * *} \\
(0.000000193)\end{array}$ & $\begin{array}{r}0.000000513^{* * *} \\
(0.000000050)\end{array}$ & $\begin{array}{r}0.000000478^{* * * *} \\
(0.000000065)\end{array}$ & $\begin{array}{r}0.00000123 * * * \\
(0.000000138)\end{array}$ \\
\hline Industry & $\begin{array}{r}0.000000011^{* * * *} \\
(0.000000001)\end{array}$ & $\begin{array}{r}0.000000024 * * * \\
(0.000000005)\end{array}$ & $\begin{array}{r}0.000000104^{* * * *} \\
(0.000000030)\end{array}$ & $\begin{array}{r}0.000000047^{* * * *} \\
(0.000000003)\end{array}$ & $\begin{array}{r}0.000000003 \\
(0.000000002)\end{array}$ & $\begin{array}{r}0.000000061 * * * \\
(0.000000013)\end{array}$ & $\begin{array}{r}0.000000049 * * * \\
(0.000000014)\end{array}$ & $\begin{array}{r}0.000000079 * * * \\
(0.000000030)\end{array}$ & $\begin{array}{c}0.000000031^{*} \\
(0.000000018)\end{array}$ \\
\hline No. Obs. & 1198 & 1198 & 1198 & 1198 & 1198 & 1198 & 1198 & 1198 & 1198 \\
\hline
\end{tabular}


Table B2.1. Estimation Results of Panel quantile regression with Estimated Coefficients of all Control Variables. The case of ln debt and family power.

\begin{tabular}{|c|c|c|c|c|c|c|c|c|c|}
\hline & \multicolumn{9}{|c|}{ Ln_debt } \\
\hline & quant. 25 & quant. 50 & quant. 75 & quant. 25 & quant. 50 & quant. 75 & quant. 25 & quant. 50 & quant. 75 \\
\hline Illiq & $\begin{array}{r}364.220 * * * \\
(78.133)\end{array}$ & $\begin{array}{r}46.738 \\
(84.874)\end{array}$ & $\begin{array}{r}65.480 \\
(203.167)\end{array}$ & & & & & & \\
\hline Sh_turn & & & & $\begin{array}{r}-26.942 * * * \\
(2.171)\end{array}$ & $\begin{array}{r}-8.997 * * * \\
(1.468)\end{array}$ & $\begin{array}{r}-6.879 * * * \\
(0.398)\end{array}$ & & & \\
\hline Mo_turn & & & & & & & $\begin{array}{r}-647458.9 * * * \\
(59976.92)\end{array}$ & $\begin{array}{r}-237837.000 * * * \\
(26920.1)\end{array}$ & $\begin{array}{r}-169339.7 * * * \\
(24468.8)\end{array}$ \\
\hline Fam_pow & $\begin{array}{r}-0.008^{* * * *} \\
(0.001)\end{array}$ & $\begin{array}{r}-0.012 * * * \\
(0.001)\end{array}$ & $\begin{array}{r}-0.007 * * * \\
(0.001)\end{array}$ & $\begin{array}{r}-0.012 * * * \\
(0.002)\end{array}$ & $\begin{array}{r}-0.004 * * * \\
(0.000)\end{array}$ & $\begin{array}{r}-0.003 * * * \\
(0.000)\end{array}$ & $\begin{array}{r}-0.010^{* * *} \\
(0.000)\end{array}$ & $\begin{array}{r}-0.006^{* * *} \\
(0.000)\end{array}$ & $\begin{array}{r}-0.002 * * \\
(0.001)\end{array}$ \\
\hline $\begin{array}{l}\text { Fam_pow* } \\
\text { illiq_ }\end{array}$ & $\begin{array}{r}-63.641 * * \\
(27.902)\end{array}$ & $\begin{array}{l}-44.792^{*} \\
(23.253)\end{array}$ & $\begin{array}{r}-173.788 * * * \\
(49.735)\end{array}$ & & & & & & \\
\hline $\begin{array}{l}\text { Fam_pow* } \\
\text { sh_turn }\end{array}$ & & & & $\begin{array}{r}3.760 * * * \\
(0.570)\end{array}$ & $\begin{array}{r}2.425 * * * \\
(0.580)\end{array}$ & $\begin{array}{r}1.522 * * * \\
(0.165)\end{array}$ & & & \\
\hline $\begin{array}{l}\text { Fam_pow* } \\
\text { mo_turn }\end{array}$ & & & & & & & $\begin{array}{r}103628.2 * * * \\
(7157.584)\end{array}$ & $\begin{array}{r}72901.55^{* * * *} \\
(10880.65)\end{array}$ & $\begin{array}{r}26467.53 * * * \\
(4963.908)\end{array}$ \\
\hline Firm_size & $\begin{array}{r}1.119 * * * \\
(0.019)\end{array}$ & $\begin{array}{r}1.171 * * * \\
(0.005)\end{array}$ & $\begin{array}{r}1.271 * * * \\
(0.017)\end{array}$ & $\begin{array}{r}1.130 * * * \\
(0.005)\end{array}$ & $\begin{array}{r}1.098^{* * *} \\
(0.003)\end{array}$ & $\begin{array}{r}1.109 * * * \\
(0.002)\end{array}$ & $\begin{array}{r}1.133 * * * \\
(0.007)\end{array}$ & $\begin{array}{r}1.084 * * * \\
(0.001)\end{array}$ & $\begin{array}{r}1.063^{* * *} \\
(0.006)\end{array}$ \\
\hline Market_book & $\begin{array}{c}-0.000^{*} \\
(0.000)\end{array}$ & $\begin{array}{r}-0.000 * * * \\
(0.000)\end{array}$ & $\begin{array}{r}0.000 * * \\
(0.000)\end{array}$ & $\begin{array}{r}-0.000 \\
(0.000)\end{array}$ & $\begin{array}{r}-0.000 * * * \\
(0.000)\end{array}$ & $\begin{array}{r}-0.000 \\
(0.000)\end{array}$ & $\begin{array}{r}-0.000 \\
(0.000)\end{array}$ & $\begin{array}{r}-0.000 * * * \\
(0.000)\end{array}$ & $\begin{array}{r}-0.000 \\
(0.000)\end{array}$ \\
\hline Profit & $\begin{array}{r}-1.849 * * * \\
(0.616)\end{array}$ & $\begin{array}{r}-0.487 * * * \\
(0.109)\end{array}$ & $\begin{array}{r}-1.064 * * * \\
(0.335)\end{array}$ & $\begin{array}{r}-2.018^{* * *} \\
(0.085)\end{array}$ & $\begin{array}{r}-2.291^{* * *} \\
(0.051)\end{array}$ & $\begin{array}{r}-1.991 * * * \\
(0.047)\end{array}$ & $\begin{array}{r}-2.287 * * * \\
(0.042)\end{array}$ & $\begin{array}{r}-2.374 * * * \\
(0.016)\end{array}$ & $\begin{array}{r}-2.169 * * * \\
(0.119)\end{array}$ \\
\hline Tang_ass & $\begin{array}{r}0.831 * * * \\
(0.082)\end{array}$ & $\begin{array}{r}0.993 * * * \\
(0.060)\end{array}$ & $\begin{array}{r}1.000 * * * \\
(0.088)\end{array}$ & $\begin{array}{r}0.333 * * * \\
(0.032)\end{array}$ & $\begin{array}{r}0.082 * * * \\
(0.017)\end{array}$ & $\begin{array}{r}0.231 * * * \\
(0.020)\end{array}$ & $\begin{array}{r}0.309 * * * \\
(0.015)\end{array}$ & $\begin{array}{r}0.129 * * * \\
(0.038)\end{array}$ & $\begin{array}{r}-0.043 \\
(0.060)\end{array}$ \\
\hline Depre_ass & $\begin{array}{r}0.772 * * * \\
(0.056)\end{array}$ & $\begin{array}{r}1.093 * * * \\
(0.057)\end{array}$ & $\begin{array}{r}1.110 * * * \\
(0.093)\end{array}$ & $\begin{array}{r}0.684 * * * \\
(0.072)\end{array}$ & $\begin{array}{r}0.251 * * * \\
(0.010)\end{array}$ & $\begin{array}{r}0.259 * * * \\
(0.011)\end{array}$ & $\begin{array}{r}0.542 * * * \\
(0.011)\end{array}$ & $\begin{array}{r}0.270 * * * \\
(0.012)\end{array}$ & $\begin{array}{l}0.060^{*} \\
(0.034)\end{array}$ \\
\hline Industry & $\begin{array}{r}-0.009 \\
(0.013)\end{array}$ & $\begin{array}{r}-0.027 * * * \\
(0.003)\end{array}$ & $\begin{array}{r}-0.013 \\
(0.012)\end{array}$ & $\begin{array}{r}-0.005 \\
(0.003)\end{array}$ & $\begin{array}{r}0.013 * * * \\
(0.004)\end{array}$ & $\begin{array}{r}0.005 * * * \\
(0.001)\end{array}$ & $\begin{array}{r}0.006^{* * *} \\
(0.002)\end{array}$ & $\begin{array}{r}0.005 * * * \\
(0.001)\end{array}$ & $\begin{array}{r}-0.008 \\
(0.015)\end{array}$ \\
\hline No. Obs. & 1198 & 1198 & 1198 & 1198 & 1198 & 1198 & 1198 & 1198 & 1198 \\
\hline
\end{tabular}


Table B2.2. Estimation Results of Panel quantile regression with Estimated Coefficients of all Control Variables. The case of market leverage and family power.

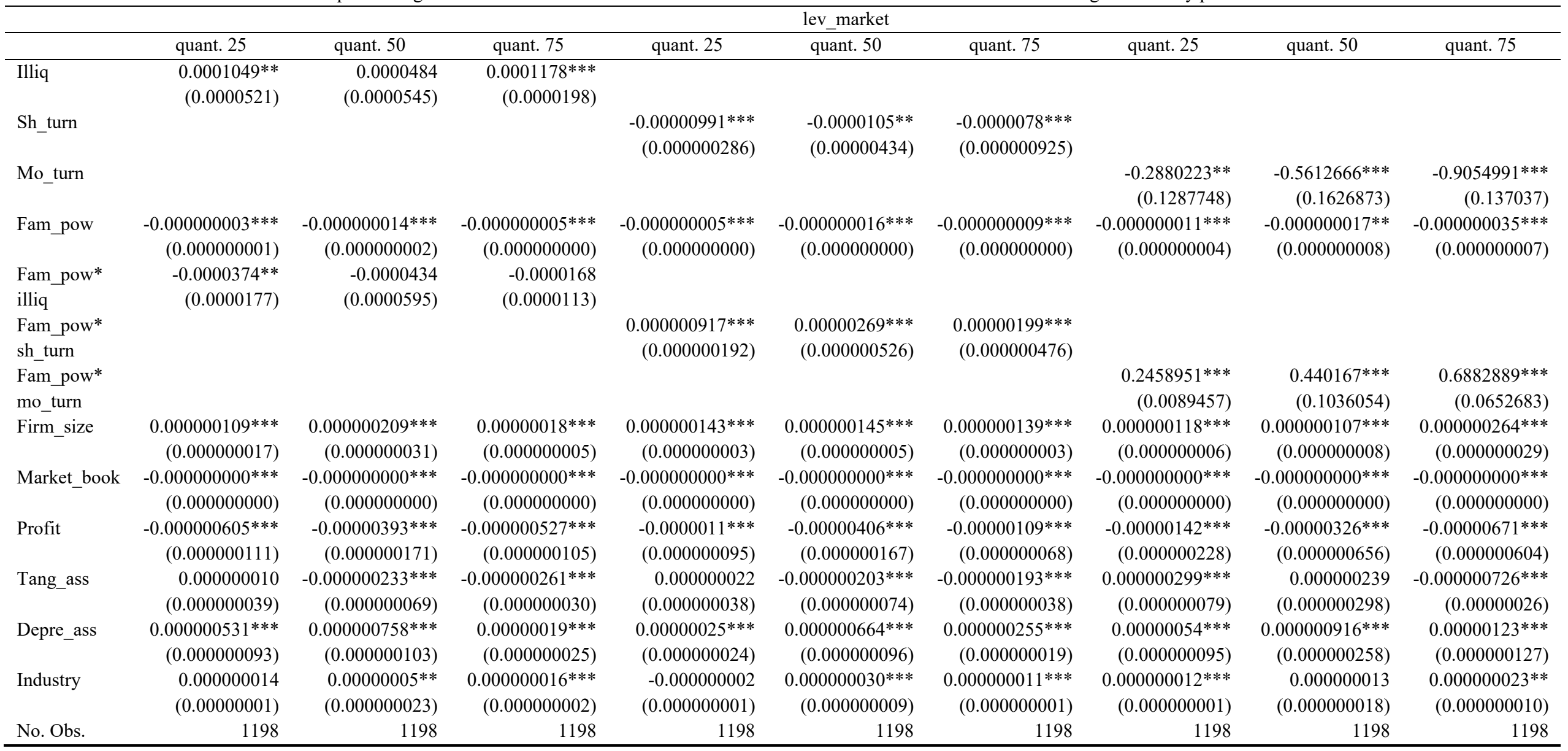


Table B3.1. Estimation Results of Panel quantile regression with Estimated Coefficients of all Control Variables. The case of ln debt and family voting power.

\begin{tabular}{|c|c|c|c|c|c|c|c|c|c|}
\hline & & & & & lev_lndebt & & & & \\
\hline & quant. 25 & quant. 50 & quant. 75 & quant. 25 & quant. 50 & quant. 75 & quant. 25 & quant. 50 & quant. 75 \\
\hline Illiq & $\begin{array}{r}185.938 * * * \\
(48.209)\end{array}$ & $\begin{array}{r}391.476^{* * *} \\
(150.388)\end{array}$ & $\begin{array}{r}464.513 * * * \\
(108.176)\end{array}$ & & & & & & \\
\hline Sh_turn & & & & $\begin{array}{r}1.330 \\
(2.787)\end{array}$ & $\begin{array}{r}-489236.1 * * * \\
(49067.09)\end{array}$ & $\begin{array}{r}-6.517 * * \\
(2.578)\end{array}$ & & & \\
\hline Mo_turn & & & & & & & $\begin{array}{r}-489236.1^{* * * *} \\
(49067.09)\end{array}$ & $\begin{array}{r}-124508.2 * * * \\
(9538.592)\end{array}$ & $\begin{array}{r}-31340.62 * * \\
(13855.17)\end{array}$ \\
\hline Fam_percent & $\begin{array}{r}-0.091 * * \\
(0.045)\end{array}$ & $\begin{array}{r}0.078 \\
(0.081)\end{array}$ & $\begin{array}{r}0.024 \\
(0.056)\end{array}$ & $\begin{array}{r}-0.450^{* * * *} \\
(0.079)\end{array}$ & $\begin{array}{r}-0.060 \\
(0.094)\end{array}$ & $\begin{array}{r}-0.017 \\
(0.028)\end{array}$ & $\begin{array}{r}-0.060 \\
(0.094)\end{array}$ & $\begin{array}{r}-0.156^{* * *} \\
(0.015)\end{array}$ & $\begin{array}{r}-0.245 * * * \\
(0.031)\end{array}$ \\
\hline $\begin{array}{l}\text { Fam_percent* } \\
\text { illiq }\end{array}$ & $\begin{array}{r}-1063.000 \\
(688.557)\end{array}$ & $\begin{array}{r}-3611.903 * * * \\
(1079.296)\end{array}$ & $\begin{array}{r}-4738.679 * * * \\
(1831.787)\end{array}$ & & & & & & \\
\hline $\begin{array}{l}\text { Fam_pecent* } \\
\text { sh_turn }\end{array}$ & & & & $\begin{array}{r}62.131 * * * \\
(5.494)\end{array}$ & $\begin{array}{r}79167.78 \\
(116323.5)\end{array}$ & $\begin{array}{r}27.151 \\
(21.671)\end{array}$ & & & \\
\hline $\begin{array}{l}\text { Fam_percent* } \\
\text { mo_turn }\end{array}$ & & & & & & & $\begin{array}{r}79167.78 \\
(116323.5)\end{array}$ & $\begin{array}{r}914429.8^{* * *} \\
(17280.02)\end{array}$ & $\begin{array}{r}1057921.000 * * * \\
(60616.99)\end{array}$ \\
\hline Firm_size & $\begin{array}{r}1.171 * * * \\
(0.003)\end{array}$ & $\begin{array}{r}1.243 * * * \\
(0.016)\end{array}$ & $\begin{array}{r}1.248^{* * * *} \\
(0.010)\end{array}$ & $\begin{array}{r}1.224 * * * \\
(0.007)\end{array}$ & $\begin{array}{r}1.175 * * * \\
(0.014)\end{array}$ & $\begin{array}{r}1.138 * * * \\
(0.013)\end{array}$ & $\begin{array}{r}1.175^{* * *} \\
(0.014)\end{array}$ & $\begin{array}{r}1.090^{* * *} \\
(0.001)\end{array}$ & $\begin{array}{r}1.080^{* * *} \\
(0.001)\end{array}$ \\
\hline Market_book & $\begin{array}{r}0.000 * * * \\
(0.000)\end{array}$ & $\begin{array}{r}-0.000 \\
(0.000)\end{array}$ & $\begin{array}{r}-0.000 * * * \\
(0.000)\end{array}$ & $\begin{array}{r}0.000^{* * *} \\
(0.000)\end{array}$ & $\begin{array}{l}0.000^{*} \\
(0.000)\end{array}$ & $\begin{array}{r}0.000 \\
(0.000)\end{array}$ & $\begin{array}{l}0.000^{*} \\
(0.000)\end{array}$ & $\begin{array}{r}-0.000^{* * * *} \\
(0.000)\end{array}$ & $\begin{array}{r}0.000^{* * *} \\
(0.000)\end{array}$ \\
\hline Profit & $\begin{array}{r}-1.239 * * * \\
(0.063)\end{array}$ & $\begin{array}{r}-0.645^{*} \\
(0.386)\end{array}$ & $\begin{array}{r}-1.429 * * * \\
(0.124)\end{array}$ & $\begin{array}{r}-1.642 * * * \\
(0.110)\end{array}$ & $\begin{array}{r}-1.896^{* * * *} \\
(0.146)\end{array}$ & $\begin{array}{r}-1.207 * * * \\
(0.244)\end{array}$ & $\begin{array}{r}-1.896 * * * \\
(0.146)\end{array}$ & $\begin{array}{r}-2.422 * * * \\
(0.016)\end{array}$ & $\begin{array}{r}-2.376^{* * * *} \\
(0.044)\end{array}$ \\
\hline Tang_ass & $\begin{array}{r}0.826 * * * \\
(0.023)\end{array}$ & $\begin{array}{r}0.570 * * * \\
(0.046)\end{array}$ & $\begin{array}{r}0.444 * * * \\
(0.133)\end{array}$ & $\begin{array}{r}0.669^{* * *} \\
(0.013)\end{array}$ & $\begin{array}{r}0.672 * * * \\
(0.053)\end{array}$ & $\begin{array}{r}0.160 * * * \\
(0.059)\end{array}$ & $\begin{array}{r}0.672 * * * \\
(0.053)\end{array}$ & $\begin{array}{r}0.078 * * * \\
(0.007)\end{array}$ & $\begin{array}{r}0.123 * * * \\
(0.010)\end{array}$ \\
\hline Depre_ass & $\begin{array}{r}1.007 * * * \\
(0.051)\end{array}$ & $\begin{array}{r}1.015 * * * \\
(0.081)\end{array}$ & $\begin{array}{r}0.680 * * * \\
(0.089)\end{array}$ & $\begin{array}{r}0.328^{* * *} \\
(0.014)\end{array}$ & $\begin{array}{r}0.545^{* * *} \\
(0.110)\end{array}$ & $\begin{array}{r}0.871 * * * \\
(0.131)\end{array}$ & $\begin{array}{r}0.545^{* * *} \\
(0.110)\end{array}$ & $\begin{array}{r}0.243 * * * \\
(0.004)\end{array}$ & $\begin{array}{r}0.404 * * * \\
(0.021)\end{array}$ \\
\hline Industry & $\begin{array}{r}0.024 * * * \\
(0.002)\end{array}$ & $\begin{array}{r}0.023 * * * \\
(0.006)\end{array}$ & $\begin{array}{r}0.020 * * * \\
(0.002)\end{array}$ & $\begin{array}{r}0.029 * * * \\
(0.002)\end{array}$ & $\begin{array}{r}-0.015^{* * *} \\
(0.003)\end{array}$ & $\begin{array}{r}-0.003 \\
(0.008)\end{array}$ & $\begin{array}{r}-0.015^{* * *} \\
(0.003)\end{array}$ & $\begin{array}{r}-0.009 * * * \\
(0.000)\end{array}$ & $\begin{array}{r}0.011 * * * \\
(0.001)\end{array}$ \\
\hline No. Obs. & 1198 & 1198 & 1198 & 1198 & 1198 & 1198 & 1198 & 1198 & 1198 \\
\hline
\end{tabular}


Table B3.2. Estimation Results of Panel quantile regression with Estimated Coefficients of all Control Variables. The case of market leverage and family voting power

\begin{tabular}{|c|c|c|c|c|c|c|c|c|c|}
\hline & \multicolumn{9}{|c|}{ lev_market } \\
\hline & quant. 25 & quant. 50 & quant. 75 & quant. 25 & quant. 50 & quant. 75 & quant. 25 & quant. 50 & quant. 75 \\
\hline Illiq & $\begin{array}{r}0.0001544 * * * \\
(0.0000569)\end{array}$ & $\begin{array}{r}0.0005065^{* * * *} \\
(0.0001774)\end{array}$ & $\begin{array}{r}0.0004127 * * \\
(0.0002042)\end{array}$ & & & & & & \\
\hline Sh_turn & & & & $\begin{array}{r}-0.00000593 * * * \\
(0.000000725)\end{array}$ & $\begin{array}{r}0.000000027 \\
(0.0000054)\end{array}$ & $\begin{array}{r}-0.000037 * * * \\
(0.00000481)\end{array}$ & & & \\
\hline Mo_turn & & & & & & & $\begin{array}{r}-0.0159943 \\
(0.050114)\end{array}$ & $\begin{array}{r}-0.3331633 * * * \\
(0.1179319)\end{array}$ & $\begin{array}{r}-0.3204377 \\
(0.3120048)\end{array}$ \\
\hline Fam_percent & $\begin{array}{r}-0.000000382 * * * \\
(0.000000116)\end{array}$ & $\begin{array}{r}-0.000000132 \\
(0.000000134)\end{array}$ & $\begin{array}{r}-0.000000231 * * * \\
(0.000000062)\end{array}$ & $\begin{array}{l}-0.000000195 \\
(0.00000026)\end{array}$ & $\begin{array}{c}-0.000000398^{*} \\
(0.000000215)\end{array}$ & $\begin{array}{r}-0.000000871^{* *} \\
(0.000000403)\end{array}$ & $\begin{array}{r}-0.000000345^{* *} \\
(0.000000161)\end{array}$ & $\begin{array}{r}-0.000000171 \\
(0.000000371)\end{array}$ & $\begin{array}{r}-0.000000376^{*} \\
(0.000000225)\end{array}$ \\
\hline $\begin{array}{l}\text { Fam_percent* } \\
\text { illiq }\end{array}$ & $\begin{array}{r}-0.0020765^{* *} \\
(0.0008846)\end{array}$ & $\begin{array}{r}-0.0003697 \\
(0.0012941)\end{array}$ & $\begin{array}{r}-0.0065976^{* * * *} \\
(0.0015043)\end{array}$ & & & & & & \\
\hline $\begin{array}{l}\text { Fam_pecent* } \\
\text { sh_turn }\end{array}$ & & & & $\begin{array}{l}0.0000163 \\
(0.000017)\end{array}$ & $\begin{array}{r}0.0000405^{* *} \\
(0.0000172)\end{array}$ & $\begin{array}{r}0.0000132 \\
(0.0000242)\end{array}$ & & & \\
\hline $\begin{array}{l}\text { Fam_percent* } \\
\text { mo_turn }\end{array}$ & & & & & & & $\begin{array}{r}0.182238 \\
(0.1933603)\end{array}$ & $\begin{array}{r}0.7587926 \\
(0.7193139)\end{array}$ & $\begin{array}{r}0.6374409 * \\
(0.3756624)\end{array}$ \\
\hline Firm_size & $\begin{array}{r}0.000000158^{* * * *} \\
(0.000000006)\end{array}$ & $\begin{array}{r}0.000000115^{* * * *} \\
(0.000000003)\end{array}$ & $\begin{array}{r}0.000000149 * * * \\
(0.000000034)\end{array}$ & $\begin{array}{r}0.000000067^{* *} \\
(0.00000003)\end{array}$ & $\begin{array}{r}0.000000163^{* * * *} \\
(0.000000035)\end{array}$ & $\begin{array}{r}0.000000307 * * * \\
(0.000000020)\end{array}$ & $\begin{array}{r}0.000000101 * * * \\
(0.000000006)\end{array}$ & $\begin{array}{r}0.00000025^{* * *} \\
(0.000000090)\end{array}$ & $\begin{array}{r}0.000000444 * * * \\
(0.000000054)\end{array}$ \\
\hline Market_book & $\begin{array}{r}-0.000000000 * * * \\
(0.000000000)\end{array}$ & $\begin{array}{r}-0.000000000 * * * \\
(0.000000000)\end{array}$ & $\begin{array}{r}-0.000000000^{* * * *} \\
(0.000000000)\end{array}$ & $\begin{array}{r}-0.000000000 * * * \\
(0.000000000)\end{array}$ & $\begin{array}{r}-0.000000000 * * * \\
(0.000000000)\end{array}$ & $\begin{array}{r}-0.000000000 * * * \\
(0.000000000)\end{array}$ & $\begin{array}{r}-0.000000000 * * * \\
(0.000000000)\end{array}$ & $\begin{array}{r}-0.000000000 * * * \\
(0.000000000)\end{array}$ & $\begin{array}{r}-0.000000000^{* * * *} \\
(0.000000000)\end{array}$ \\
\hline Profit & $\begin{array}{r}-0.000000507 * * * \\
(0.000000179)\end{array}$ & $\begin{array}{r}-0.00000424 * * * \\
(0.000000154)\end{array}$ & $\begin{array}{r}-0.00000248^{* * *} \\
(0.00000044)\end{array}$ & $\begin{array}{r}-0.000000326 \\
(0.000000332)\end{array}$ & $\begin{array}{r}-0.00000296^{* * *} \\
(0.000000536)\end{array}$ & $\begin{array}{r}-0.00000495^{* * *} \\
(0.000000477)\end{array}$ & $\begin{array}{r}-0.000000831 * * * \\
(0.000000064)\end{array}$ & $\begin{array}{r}-0.00000379 * * * \\
(0.000000556)\end{array}$ & $\begin{array}{r}-0.00000438^{* * * *} \\
(0.000000566)\end{array}$ \\
\hline Tang_ass & $\begin{array}{r}0.000000144 \\
(0.000000090)\end{array}$ & $\begin{array}{r}-0.000000125 \\
(0.00000012)\end{array}$ & $\begin{array}{r}0.000000079 \\
(0.000000143)\end{array}$ & $\begin{array}{r}0.000000113 \\
(0.000000097)\end{array}$ & $\begin{array}{r}0.00000015 \\
(0.000000171)\end{array}$ & $\begin{array}{r}-0.000000638^{* * * *} \\
(0.00000011)\end{array}$ & $\begin{array}{r}0.000000074 \\
(0.000000046)\end{array}$ & $\begin{array}{r}0.000000005 \\
(0.000000211)\end{array}$ & $\begin{array}{r}-0.000000075 \\
(0.000000287)\end{array}$ \\
\hline Depre_ass & $\begin{array}{r}0.000000415^{* * *} \\
(0.000000044)\end{array}$ & $\begin{array}{r}0.000000331^{* * *} \\
(0.000000026)\end{array}$ & $\begin{array}{r}0.000000865^{* * *} \\
(0.000000095)\end{array}$ & $\begin{array}{r}0.000000419^{* * *} \\
(0.000000102)\end{array}$ & $\begin{array}{r}0.00000121 * * * \\
(0.000000195)\end{array}$ & $\begin{array}{r}0.00000124 * * * \\
(0.000000070)\end{array}$ & $\begin{array}{r}0.000000426^{* * *} \\
(0.000000088)\end{array}$ & $\begin{array}{r}0.00000108^{*} \\
(0.000000635)\end{array}$ & $\begin{array}{r}0.00000177^{* * *} * \\
(0.000000269)\end{array}$ \\
\hline Industry & $\begin{array}{r}0.000000018^{* * *} * \\
(0.000000005)\end{array}$ & $\begin{array}{r}0.000000022^{* *} \\
(0.000000010)\end{array}$ & $\begin{array}{r}-0.000000018^{* *} \\
(0.000000009)\end{array}$ & $\begin{array}{r}0.000000014 * * * \\
(0.000000003)\end{array}$ & $\begin{array}{r}0.000000063^{* * *} \\
(0.000000018)\end{array}$ & $\begin{array}{r}0.000000009 \\
(0.000000017)\end{array}$ & $\begin{array}{r}0.000000030 * * * \\
(0.000000011)\end{array}$ & $\begin{array}{r}0.000000052 \\
(0.000000039)\end{array}$ & $\begin{array}{r}0.000000238^{* * * *} \\
(0.000000037)\end{array}$ \\
\hline No. Obs. & 1198 & 1198 & 1198 & 1198 & 1198 & 1198 & 1198 & 1198 & 1198 \\
\hline
\end{tabular}




\section{References}

Amihud, Y. (2002). Illiquidity and stock returns: cross-section and time-series effects. Journal of Financial Markets, 5(1), 31-56.

Baker, M., \& Wurgler, J. (2002). Market timing and capital structure. The Journal of Finance, $57(1), 1-32$.

Bianco, M., \& Nicodano, G. (2006). Pyramidal groups and debt. European Economic Review, 50(4), 937-961.

Butler, A. W., Grullon, G., \& Weston, J. P. (2005). Stock market liquidity and the cost of issuing equity. Journal of Financial Quantitative Analysis, 40(2), 331-348.

Canay, I. A. (2011). A simple approach to quantile regression for panel data. The Econometrics Journal, 14(3), 368-386.

Céspedes, J., González, M., \& Molina, C. A. (2010). Ownership and capital structure in Latin America. Journal of Business Research, 63(3), 248-254.

Dang, T. L., Ly Ho, H., Dzung Lam, C., Thao Tran, T., \& Vo, X. V. (2019). Stock liquidity and capital structure: International evidence. Cogent Economics \& Finance, 7(1), 1587804.

Deesomsak, R., Paudyal, K., \& Pescetto, G. (2004). The determinants of capital structure: evidence from the Asia Pacific region. Journal of Multinational Financial Management, 14(4-5), 387-405.

Duc, H. V., \& Tri, M. N. (2014). The impact of corporate governance on firm performance: Empirical study in Vietnam. International Journal of Economics and Finance, 6(6), 1-13.

ElBannan, M. A. (2017). Stock market liquidity, family ownership, and capital structure choices in an emerging country. Emerging Markets Review.

Fama, E. F., \& French, K. R. (2002). Testing trade-off and pecking order predictions about dividends and debt. The Review of Financial Studies, 15(1), 1-33.

Frieder, L., \& Martell, R. (2006). On capital structure and the liquidity of a firm's stock.

Gómez-Mejía, L. R., Haynes, K. T., Núñez-Nickel, M., Jacobson, K. J., \& Moyano-Fuentes, J. (2007). Socioemotional wealth and business risks in family-controlled firms: Evidence from Spanish olive oil mills. Administrative science quarterly, 52(1), 106-137.

Gomez-Mejia, L. R., Larraza-Kintana, M., \& Makri, M. (2003). The determinants of executive compensation in family-controlled public corporations. Academy of Management Journal, 46(2), 226-237.

Gomez-Mejia, L. R., Nunez-Nickel, M., \& Gutierrez, I. (2001). The role of family ties in agency contracts. Academy of Management Journal, 44(1), 81-95.

Hennessy, C. A., \& Whited, T. M. (2005). Debt dynamics. Journal of Finance, 60(3), 11291165.

Kien, S. N. (2020). Volatility and Specific Risk Toward Family's Performance in an Emerging Country. Asia-Pacific Financial Markets, 27(3), 363-386. doi:10.1007/s10690-01909297-x

Kien, S. N., \& Duc, H. V. (2015). Family Ownership in Vietnam's Listed Firms: Does It Really Matter? Paper presented at the The Eighth Vietnam Economists Annual Meeting.

King, M. R., \& Santor, E. (2008). Family values: Ownership structure, performance and capital structure of Canadian firms. Journal of Banking Finance, 32(11), 2423-2432.

Koenker, R. (2004). Quantile regression for longitudinal data. Journal of Multivariate Analysis, 91(1), 74-89.

Kryzanowski, L., Lazrak, S., \& Rakita, I. (2010). Behavior of liquidity and returns around Canadian seasoned equity offerings. Journal of Banking Finance, 34(12), 2954-2967. 
Lamarche, C. (2010). Robust penalized quantile regression estimation for panel data. Journal of Econometrics, 157(2), 396-408.

Lev, B. (1988). Toward a theory of equitable and efficient accounting policy. Accounting Review, 1-22.

Lipson, M. L., \& Mortal, S. (2009). Liquidity and capital structure. Journal of Financial Markets, 12(4), 611-644.

Miller, M. H., \& Rock, K. (1985). Dividend policy under asymmetric information. The Journal of Finance, 40(4), 1031-1051.

Modigliani, F., \& Miller, M. H. (1958). The cost of capital, corporation finance and the theory of investment. The American economic review, 48(3), 261-297.

Moussa, A. A., \& Elgiziry, K. (2019). The impact of family involvement in business on capital structure decisions: a literature review. Investment Management \& Financial Innovations, $16(1), 258$.

Myers, S. C., \& Majluf, N. S. (1984). Corporate financing and investment decisions when firms have information that investors do not have. Journal of financial economics, 13(2), 187221.

Nguyen, K. S. (2019). Volatility and Specific Risk Toward Family's Performance in an Emerging Country. Asia-Pacific Financial Markets, 1-24.

Powell, D. (2016). Quantile regression with nonadditive fixed effects. Quantile Treatment Effects, 1-28.

Setia-Atmaja, L., Tanewski, G. A., \& Skully, M. (2009). The role of dividends, debt and board structure in the governance of family controlled firms. Journal of Business Finance Accounting, 36(7-8), 863-898.

Sharma, P., \& Paul, S. (2015). Does liquidity determine capital structure? Evidence from India. Global Business Review, 16(1), 84-95.

Sirmon, D. G., Arregle, J. L., Hitt, M. A., \& Webb, J. W. (2008). The role of family influence in firms' strategic responses to threat of imitation. Entrepreneurship Theory and Practice, 32(6), 979-998.

Stulz, R. (1988). Managerial control of voting rights: Financing policies and the market for corporate control. Journal of financial economics, 20, 25-54.

Thanh, L., \& Mai, H. (2017). Determinants of capital structure: An empirical study on Vietnamese listed firms. Serbian Journal of Management, 12(1), 77-92. doi:10.5937/sjm12-10187

Tran, L. T. H., Hoang, T. T. P., \& Tran, H. X. (2018). Stock liquidity and ownership structure during and after the 2008 Global Financial Crisis: Empirical evidence from an emerging market. Emerging Markets Review, 37, 114-133.

Udomsirikul, P., Jumreornvong, S., \& Jiraporn, P. (2011). Liquidity and capital structure: The case of Thailand. Journal of Multinational Financial Management, 21(2), 106-117.

Vo, X. V. (2017). Determinants of capital structure in emerging markets: Evidence from Vietnam. Research in International Business and Finance, 40, 105-113. 